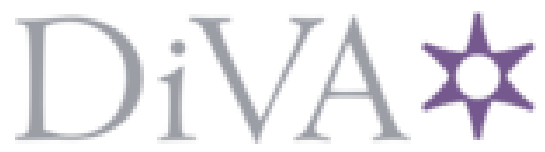

http://www.diva-portal.org

This is the published version of a paper presented at SAI Computing Conference (SAI), JUL 13-15, 2016, London, ENGLAND.

Citation for the original published paper:

Kusetogullari, H., Yavariabdi, A. (2016)

Self-Adaptive Hybrid PSO-GA Method for Change Detection Under Varying Contrast Conditions in Satellite Images.

In: Proceedings of the 2016 SAI Computing Conference (SAI) (pp. 361-368). IEEE

N.B. When citing this work, cite the original published paper.

Permanent link to this version:

http://urn.kb.se/resolve?urn=urn:nbn:se:bth-13683 


\section{Self-Adaptive Hybrid PSO-GA Method for Change Detection Under Varying Contrast Conditions in Satellite Images}

\author{
Huseyin Kusetogullari \\ Department of Computer Science and Engineering \\ Blekinge Institute of Technology \\ Karlskrona, Sweden \\ Email: hku@bth.se
}

\author{
Amir Yavariabdi \\ Department of Mechatronics Engineering \\ KTO Karatay University \\ Konya, Turkey \\ Email: amir.yavariabdi@gmail.com
}

\begin{abstract}
This paper proposes a new unsupervised satellite change detection method, which is robust to illumination changes. To achieve this, firstly, a preprocessing strategy is used to remove illumination artifacts and results in less false detection than traditional threshold-based algorithms. Then, we use the corrected input data to define a new fitness function based on the difference image. The purpose of using Self-Adaptive Hybrid Particle Swarm Optimization-Genetic Algorithm (SAPSOGA) is to combine two meta-heuristic optimization algorithms to search and find the feasible solution in the NP-hard change detection problem rapidly and efficiently. The hybrid algorithm is employed by letting the GA and PSO run simultaneously and similarities of GA and PSO have been considered to implement the algorithm, i.e. the population. In the SAPSOGA employed, in each iteration/generation the two population based algorithms share different amount of information or individual(s) between themselves. Thus, each algorithm informs each other about their best optimum results (fitness values and solution representations) which are obtained in their own population. The fitness function is minimized by using binary based SAPSOGA approach to produce binary change detection masks in each iteration to obtain the optimal change detection mask between two multi temporal multi spectral landsat images. The proposed approach effectively optimizes the change detection problem and finds the final change detection mask.
\end{abstract}

Keywords-Remote sensing; Image processing; Optimization; Self-adaptive hybrid algorithm; Genetic algorithm; Binary particle swarm optimization

\section{INTRODUCTION}

Change detection from images of the same scene captured at different time instances is of special interest in many image processing application areas such as video surveillance [1], satellite imaging [2], [3],medical image analysis [4], material analysis [5], underwater imaging [6], and driver assistance systems [7]. Remote sensing has long been used as a means of observing, monitoring, and understanding trajectories of changes in the condition of the Earth over time including natural and anthropogenic perturbation, climate change, and urbanisation. Satellite sensors are appropriate choice for these types of task as they provide a vast amount of detailed information at a specific resolution range. In remote sensing context, change detection is the process of identifying differences between corresponding objects or phenomenons by analysing the images which are captured at different times [8].
There are two mostly used approaches to find the change detection mask which are supervised and unsupervised. The supervised approach is based on a classification method, which needs a training set with multi-temporal ground truth to model changed areas [9], [10]. Manually labelling and identifying changes is a cumbersome task and it is prone to errors and highly subjective depending on the expertise of the inspector. Therefore, many methods have been presented and developed to automatically identify changed and unchanged regions between the pre-registered satellite images. The unsupervised methods directly compare multi-temporal satellite images or process the difference image using pattern recognition techniques. In this approach, there is no need for a training set. In [11], Markov Random Field (MRF) is presented to apply noiseless satellite images to obtain the change detection mask. The method employs the simulated annealing (SA) method with the posteriori probability decision criterion to solve the problem. The SA method is employed to generate a set of random binary change masks which makes the method depend on the previous binary change detection image and obtained the optimum solution by using the Gibbs sampling procedure. In Celik [2], combination of Principle Component Analysis (PCA) and k-means clustering method is applied to determine the binary change detection mask. PCA is used to compute the eigenvalues and eigenvectors which are used to partition the change and unchanged regions between two different satellite images by using k-means clustering. This method gives precise results with low computational cost. Other methods such as Fuzzy c-means [12], k-means [13] and normalised cut clustering [14] have also been also proposed to solve the change detection problem in satellite imaging. However, these methods are unable to find changed and unchanged pixels effectively. Therefore, optimisation approach is necessary to search for changed and unchanged pixels to obtain the optimal binary change mask effectively on a difference image. Celik [15] uses Genetic Algorithm (GA) to cluster changed and unchanged pixels between two satellite images by minimising a cost function based on difference image. The method successfully obtains the binary change mask on the difference image. However, in GA cluster-based change detection method, a large number of iterations is needed to find the optimal result.

All proposed supervised and unsupervised change detection methods are robust to the noise but they fail to find accurate 
change detection mask if there is a contrast or illumination artefacts between two satellite images. In this paper, a new photometric invariants unsupervised satellite change detection method is proposed which is robust to shadow, shading, highlights and specularities. To achieve this, the RGB colour space transformed into chromaticity space. Then, a cost function based on the enhanced Correlation Coefficient (CC) similarity measurement [16] of two different normalised RGB Landsat images is defined and optimised by using SAPSOGA. The method is employed to run GA and PSO simultaneously to find the weak and strong changes by updating each subpopulation in SAPSOGA, iteratively. After that, meta-heuristic algorithms share individual(s) or information between each other to increase the probability of obtaining optimum binary change mask quickly and efficiently. The main contribution of this paper are:

- $\quad$ Proposing a new optimisation approach to find binary change detection mask from two different multispectral satellite images, unlike other change detection methods which are based on two satellite images [2], [11], [12], [15].

- Adopting the SAPSOGA to find changed and unchanged pixels by minimising a new cost function.

- Reducing the influence of illumination changes between two images using a photometric invariant approach, which improves the performance of the proposed method in terms of correct detection rate.

The rest of the paper is organised as follows. In section 2, the proposed method is explained and fitness cost function is defined. The performance of the proposed method is provided in Section 3. In the last section, we conclude the paper.

\section{PROPOSED METHOD}

\section{A. Normalisation Technique}

Illumination change is one of the main reasons to cause false detections on traditional change detection methods. For instance, Fig. 1 shows the darker and lighter of satellite images of the original satellite image. Therefore, it is vital to propose a framework which is robust against illumination. In order to achieve this, RGB colours are normalised to be insensitive to brightness differences of images. This photometric invariants technique is formulated as:

$$
(R, G, B)^{\top} \mapsto\left(\frac{R}{N}, \frac{G}{N}, \frac{B}{N}\right)^{\top}
$$

where $N$ is a normalisation factor that depends on the $R$, $G$, and $B$. Note that the normalised RGB space known as chromaticity space. The most popular representative for chromaticity spaces is arithmetic mean $N=\frac{(R+G+B)}{3}$. This photometric invariants technique is very robust against shadow and shading. In the proposed method, this normalisation strategy is used as a preprocessing strategy to remove the effect of lighting geometry.

\section{B. Change Detection Problem}

Let us consider two pre-aligned and normalised RGB Landsat images, $I_{1}$ and $I_{2}$, which are acquired on the same

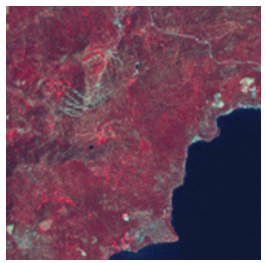

(a)

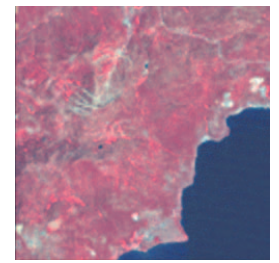

(b)

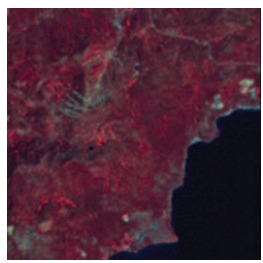

(c)
Fig. 1: Satellite images with different contrast levels. (a) Original, (b) bright, (c) low contrast.

geographical region, but at two different times. Here, the main goal is to obtain a binary map $I_{b}$, based on the CC similarity measurement computed from multi temporal multi spectral images.

Note that the binary change detection map $I_{b}$ is a binary image with size of $(H \times W) \times n$. In addition, there are $2^{(H \times W)^{n}}$ possible solutions or combinations to find the optimal change detection map. Each of these binary images has a corresponding fitness function which is computed from the improved CC similarity measurement and one of them provides the minimum fitness value which results in obtaining the optimum binary change detection map $I_{b}$. Since it is impossible to examine all possible combinations to find the minimum fitness value, it is necessary to use an optimisation strategy to find the optimal change detection map. Therefore, we uses a new cost function which is minimised by using the SAPSOGA.

\section{Self-Adaptive Hybrid Particle Swarm Optimisation-Genetic Algorithm (SAPSOGA)}

In the proposed method, binary based SAPSOGA is used to optimise the change detection problem to find the final change detection map. GA and PSO are the population-based algorithms that have similarities and diversities between each other to optimise a search space problem. In the SAPSOGA, two sub-algorithms which are GA and PSO run simultaneously and each algorithm uses its own generation process to create new individuals in each iteration as shown in Fig. 2. Note that, generation processes of GA and PSO are given with the details in [17] and [18]. Each sub-algorithm in the the SAPSOGA may need additional information if the distribution or variance of individuals is very high in their own population pool. Therefore, they have to inform each other to reduce the variance by sharing their best fitness values and representation solutions in each iteration. Thus, the quality of mutual information is increasing in the sub-algorithms to achieve the optimum result. In the SAPSOGA, there are two different cases to share or exchange the individuals between the sub-populations of simultaneously running meta-heuristic algorithms. 1) If there is a large variance in one of the sub-population of GA or PSO, the GA or PSO needs large number of iteration or substantial time to reach the optimum result. Stronger individuals are sent from one algorithm to the other algorithm. 2) If there is a small variance in both sub-populations of GA or PSO, both subalgorithms need larger number of iteration or substantial time to reach the optimum result. Best individuals are exchanged between two sub-populations. On the other hand, an algorithm 


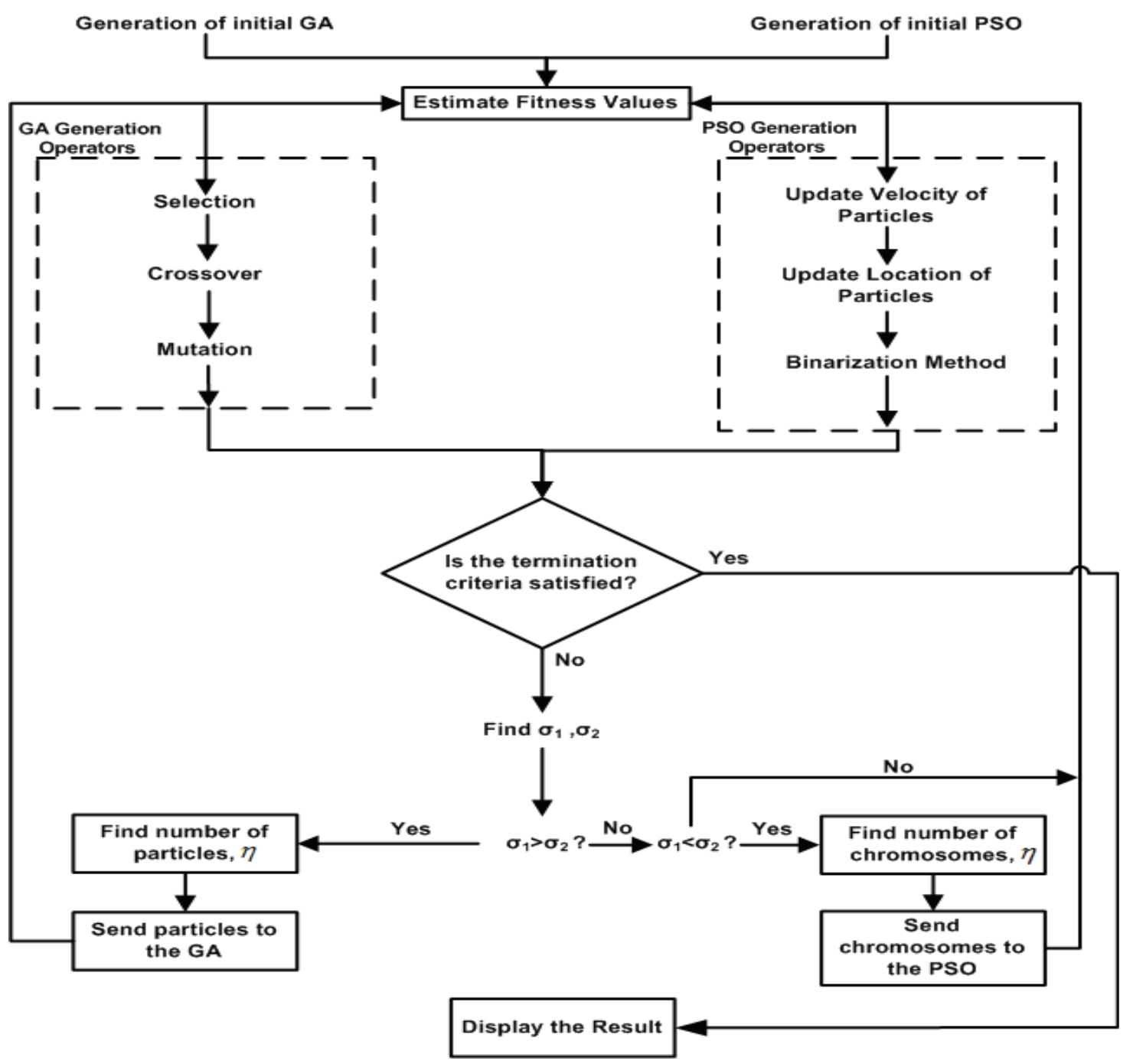

Fig. 2: Flowchart of the SAPSOGA to solve the change detection problem.

does not need information from the other algorithm in the following case: 3) If there is a small variance in a subpopulation, the GA or PSO can reach the optimum result in a short time and it does not demand information from other algorithm. In the adaptive SAPSOGA, these cases can be changeable in each iteration.

In a population, there are strong and weak individuals and strong ones have usually higher chance to obtain the optimum result faster than weak ones [19]. In the proposed method, each individual (e.g. chromosome and particle's location) is represented by a fix length array with the length of $H \times W \times n$, and each array element is assigned with a binary number. Thus, a three dimensional mask is create which includes zeros and ones to estimate the final change detection mask. Let $P c_{k 1}^{g}$ and $P p_{k 2}^{g}$ be the probability of occurrence of chromosome of $k 1$ and particle $k 2$ at generation $g$ and $C c_{k 1}^{g}$ and $C p_{k 2}^{g}$ be the fitness values in GA and PSO, respectively. In the corresponding minimisation problem, the probability of each chromosome and particle is given as:

$$
P c_{k 1}^{g}=\frac{C c_{k 1}^{g}}{\sum_{i=1}^{n_{1}} C c_{k 1}^{g}}, \quad P p_{k 1}^{g}=\frac{C p_{k 2}^{g}}{\sum_{i=1}^{n_{2}} C p_{k 2}^{g}}
$$

The mean and standard deviation of the GA $\left(\mu_{1}^{g}, \sigma_{1}^{g}\right)$ and of the PSO $\left(\mu_{2}^{g}, \sigma_{2}^{g}\right)$ are defined as follows:

$$
\begin{gathered}
\mu_{1}^{g}=\lim _{x \rightarrow m^{+}} \sum_{i=1}^{n_{1}} P c_{i}^{g}\left(C c_{i}^{g}-x\right) \\
\mu_{2}^{g}=\lim _{x \rightarrow m^{+}} \sum_{j=1}^{n_{2}} P p_{j}^{g}\left(C p_{j}^{g}-x\right), \\
\sigma_{1}^{g}=\sqrt{\sum_{i=1}^{n_{1}} P c_{i}^{g}\left(\mu_{1}^{g}-C c_{i}^{g}\right)^{2}}, \\
\sigma_{2}^{g}=\sqrt{\sum_{j=1}^{n_{2}} P p_{j}^{g}\left(\mu_{2}^{g}-C p_{j}^{g}\right)^{2}}
\end{gathered}
$$

where $m$ is defined as the convergent value. The number of individuals transferred between two sub-populations are based on the $\sigma_{1}^{g}$ and $\sigma_{2}^{g}$. If $\sigma_{1}^{g}$ and $\sigma_{2}^{g}$ are equal, then the variance in each population is similar. Therefore, there is not necessary to share information or individuals between the populations. If $\sigma_{1}^{g}$ is greater than $\sigma_{2}^{g}$, the selected weak chromosomes are replaced by the strong particles in the population of the GA, otherwise, selected weak particles are replaced by the strong chromosomes in the population of the PSO. The number of 
transferred individual $\eta$ is obtained as follows:

$$
\begin{gathered}
\eta=\sigma_{2}^{g} / \sigma_{1}^{g} * n_{1} ; \quad \text { if } \quad \sigma_{1}>\sigma_{2} \\
\eta=\sigma_{1}^{g} / \sigma_{2}^{g} * n_{2} ; \quad \text { if } \quad \sigma_{2}>\sigma_{1} \\
\eta=0 ; \quad \text { if } \quad \sigma_{1}=\sigma_{2}
\end{gathered}
$$

Each individual (e.g. chromosome and particle) in the population is represented with a fitness value which is computed by a fitness function. By finding fitness values, the strong and weak individuals can be obtained based on the fitness values and the initialised population is iteratively updated based on these individuals. The fitness function used in the SAPSOGA is defined as follows:

$$
F_{k}^{g}=\frac{1}{3} \sum_{n=1}^{3}\left[\frac{1}{2}\left(1+C_{0}^{n}\right)\right]+\left[1-\frac{1}{2}\left(1+C_{1}^{n}\right)\right]
$$

where $F_{k}^{g}$ denotes the fitness cost function of individual $k$ at iteration number $g$. Note that the correlation between unchanged pixels must be high. On the other hand, the correlation between changed pixels should be low. The correlation is estimated using the enhanced $\mathrm{CC}[16]$. The value of the $\mathrm{CC}$ [16] ranges from -1 , indicating no correlation, to 1 , indicating very highly correlation. After that, the range of the CC normalised into $[0,1]$.

The CC method [16] uses intensities in an image which are normalised with respect to the mean intensity. This makes the method invariant to bias in image intensities. Moreover, it normalises the measure with respect to image contrast by dividing the inner product of the mean-normalised intensities by the standard deviation of intensities in each image. Therefore, the $\mathrm{CC}$ approach is appropriate for comparing images which have different illumination and contrast. The CC [16] is written as:

$$
\begin{aligned}
& C_{0}^{n}=\sum_{\forall(x, y) \in B_{0}}\left(\frac{\left(I_{1}^{n}(x, y)-\bar{I}_{1, B_{0}}^{n}\right)}{s_{1,0}^{n}}-\frac{\left(I_{2}^{n}(x, y)-\bar{I}_{2, B_{0}}^{n}\right)}{s_{2,0}^{n}}\right) \\
& C_{1}^{n}=\sum_{\forall(x, y) \in B_{1}}\left(\frac{\left(I_{1}^{n}(x, y)-\bar{I}_{1, B_{1}}^{n}\right)}{s_{1,1}^{n}}-\frac{\left(I_{2}^{n}(x, y)-\bar{I}_{2, B_{1}}^{n}\right)}{s_{2,1}^{n}}\right)
\end{aligned}
$$

where $C_{0}$ and $C_{1}^{n}$ are two different fitness functions based on the changed and unchanged regions at the n-th spectral band, respectively, $B_{0}^{n}$ and $B_{1}^{n}$ denote all changed and unchanged pixels on the binary change image, respectively. $\bar{I}_{1, B_{0}}^{n}$ and $\bar{I}_{1, B_{1}}^{n}$ are the mean of $I_{1}$ estimated over changed and unchanged pixels, respectively, and similarly for $\bar{I}_{2, B_{0}}^{n}$ and $\bar{I}_{2, B_{0}}^{n}$. $s_{i, 0}^{n}$ and $s_{i, 1}^{n}$, where $i=\{1,2\}$, are the standard deviation of changed and unchanged pixels of $I_{1}$ and $I_{2}$, respectively. These are estimated as follows:

$$
\begin{aligned}
s_{i, 0}^{n} & =\sqrt{\frac{1}{N_{i, 0}^{n}} \sum_{y=1}^{H} \sum_{x=1}^{W}\left(I_{i}-\bar{I}_{i, B_{0}}^{n}\right)^{2}}, \\
s_{i, 1}^{n} & =\sqrt{\frac{1}{N_{i, 1}^{n}} \sum_{y=1}^{H} \sum_{x=1}^{W}\left(I_{i}-\bar{I}_{i, B_{1}}^{n}\right)^{2}}, \quad \text { for } \quad i=1,2
\end{aligned}
$$

where $N_{i, 0}^{n}$ and $N_{i, 1}^{n}$ are the number of changed and unchanged pixels on the change detection mask, respectively. The aim

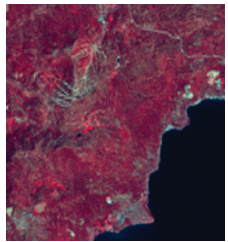

(a)

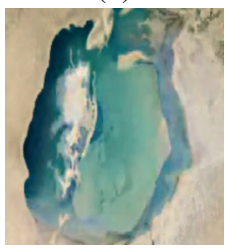

(d)

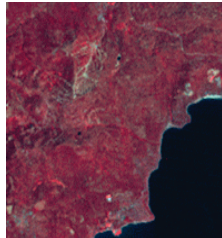

(b)

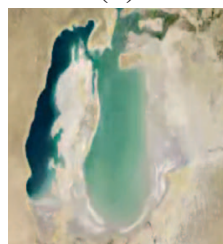

(e)

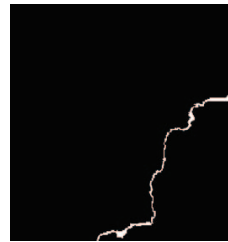

(c)

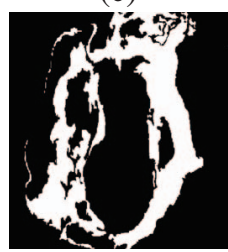

(f)
Fig. 3: The data sets used in our experiments. (a) and (b): illustration of the conifer mortality area of the Reno-Lake Tahoe in Nevada acquired on August 5, 1986 and 1992, respectively. (d) and (e): illustration of the Aral sea acquired in 1992 and 2002, respectively. (c) and (f): show the ground truth binary change maps.

of the proposed method is to optimise the cost function to find the best binary change mask which includes changes and unchanged pixels. The performance of the proposed method SAPSOGA is compared with four satellite change detection methods and simulation results verify that using preprocessing method with the SAPSOGA provides a significant performance of obtaining the final binary change detection image between two multi temporal multi spectral satellite images.

\section{EXPERIMENTAL RESULTS}

We assess the performance of our method in terms of qualitative and quantitative tests on both semi-synthetic and real multi temporal multi spectral data sets. The first data set is the area of conifer mortality of the Reno-Lake Tahoe in Nevada. The second one is the surface of the Aral sea lying between Kazakhstan in the north and Uzbekistan in the south. The data sets have been obtained from the Earth Resources Observation and Science (EROS) Centre and United States Geological Survey (USGS).

The first data [20], shown in Fig. 3 (a)-(b), is the set of Landsat images with the size of $200 \times 200$. They were captured on two different dates in 1986 and 1992. These images are used to observe the changes in the forest area for analysing and understanding the amount of drought in the area. Furthermore, ground truth binary change image of the first data is demonstrated in Fig. 3 (c)

The second data [21] includes two images with the size of $980 \times 540$. The data illustrates that the water level of Aral sea decreases in 2002 when compared to the 1992. In order to monitor and analysis the changes in the Aral sea, a region of interest with size of $500 \times 450$ is manually chosen from input images as illustrated in Fig. 3 (d)-(e). The ground truth binary change map is illustrated in Fig. 3 (f). All methods have been implemented in MATLAB and executed using Intel Core i3 processor and 3 GB RAM. In the SAPSOGA, there 
TABLE I: PARAMETERS USED IN THE SAPSOGA.

\begin{tabular}{c|c}
\hline \hline Parameter for SAPSOGA & value \\
\hline Population size $(\mathrm{P})$ & 150 \\
Mutation rate in GA $P_{m}$ & 0.1 \\
Crossover rate in GA $P_{c}$ & 0.8 \\
Two learning factors $c_{1}$ and $c_{2}$ & $2.0,2.0$ \\
Inertia weight $\omega$ & 0.5 \\
Number of generations & 20000 \\
\hline
\end{tabular}

are key parameters that affect the generation of population at each iteration and these are illustrated in Table I.

\section{A. Quantitative Error Measures}

To validate the results, three different quantitative error measures are calculated. To achieve this, the obtained change masks are compared with the corresponding ground truth images. False Alarm (FA): It is the number of unchanged pixels that are falsely flagged as changed pixels. The percentage of FA can be estimated with $P_{F A}=F A / N_{1} \times 100$, where $N_{1}$ denotes as total number of unchanged pixels in the ground truth image. Miss Alarm (MA): It is the number of changed pixels that are wrongly obtained as unchanged pixels. The percentage of MA can be estimated with $P_{M A}=M A / N_{0} \times 100$, where $N_{0}$ denotes as total number of changed pixels in the ground truth. Total Error (TE): It is the sum of the FA and MA. Thus, the percentage of TE can be estimated with $P_{T E}=(F A+M D) /\left(N_{0}+N_{1}\right) \times 100$.

\section{B. Performance Comparison of Optimisation Algorithms for Change Detection Problem}

Here, the goal is to evaluate the accuracy of our method and GA cluster-based change detection method [15]. In this manner, we understand and analyse the performance of two different search based algorithms for obtaining optimum result in change detection problem. Compared with the GA clusterbased method [15], the proposed method tends to converge to the best solution with lower iteration number. In other words, the proposed method is faster and gets better results. For instance, our method gives the best solution (binary change detection image) at iteration number 20000 but GA clusterbased method needs more than 20000 iterations to find the best result as shown in Fig. 4.

\section{Tests on Semi-synthetic Data}

The Reno-Lake Tahoe image captured in 1986 (Fig. 3 (a)) is used to generate a semi-synthetic image. This is a vital task for conducting quantitative evaluation, since the actual ground truth does not exist. To this end, firstly, the image shown in the Fig. 3 (a) is selected as a reference image $I_{1}$. After that, the intensities of $I_{1}$ in a specific region (e.g. see the mask in the Fig. 3 (c)) is changed to generate the semi-synthetic Landsat image $I_{2}$. This is achieved by:

$$
I_{2}(x, y)\left\{\begin{array}{lc}
I_{1}(x, y)-D, & \text { if } I_{1}(x, y)-D>0 \\
I_{1}(x, y)+D, & \text { otherwise }
\end{array}\right.
$$

where $(x, y) \in R O I$ and $D$ is the average of $I_{1}(x, y)$ over its $5 \times 5$ neighbourhood. This equation shows that the difference between $I_{1}$ and $I_{2}$ in the region of interest is $D$, thus the ground truth of the change map can be obtained.
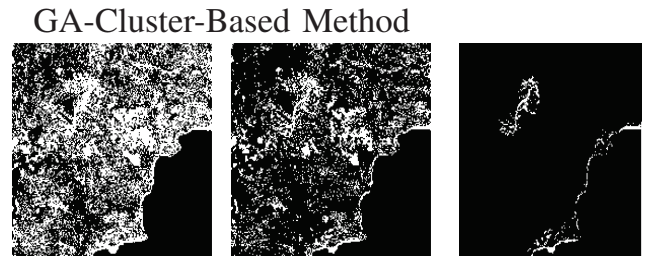

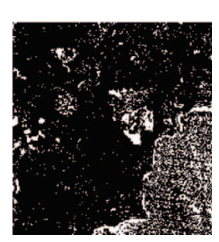

(a)

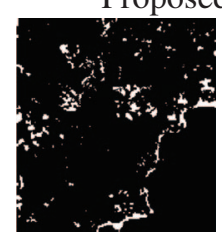

(b)

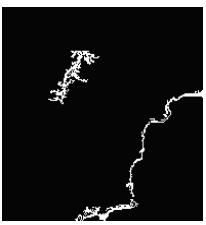

(c)

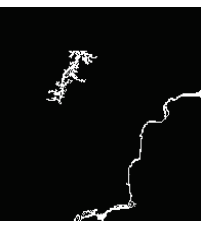

(d)
Fig. 4: Intermediate change detection masks generated by using the GA cluster-based method and the proposed method. The intermediate masks at generation (a): 40, (d): 4000, (e): 12000, and (f): 20000.

Robustness Test: Generally, during satellite image acquisition and transmission Gaussian noise is added on the signal, which superimposes random variation in signal intensity. Thus, measuring robustness of algorithms against various levels of Gaussian noise is a vital task. Hence, Peak Signal-to-Noise Ratio (PSNR), in decibel (dB), is used as a quantitative measurement for each noise level.

$$
P S N R=10 \log _{10} \frac{255^{2}}{\frac{1}{W \times H} \sum_{w=1}^{W} \sum_{h=1}^{H}\left(\left(I_{1}(w, h)-I_{2}^{\sigma}(w, h)\right)\right)^{2}}
$$

where $I_{1}$ is the reference image and $I_{2}^{\sigma}$ is a corrupted image with various levels of zero mean Gaussian noise. Note that as noise increases, the quality of image decreases and thus, PSNR decreases. After corrupting the semi-synthetic image $I_{2}^{\sigma}$ with zero mean Gaussian noise with a standard deviations $\sigma$ in the range between 0.001 and 1.0, the change detection algorithms are used to evaluate their effectiveness and robustness.

Results: Experiments on semi-synthetic data (Fig. 5 (b)) with different levels of Gaussian noise (e.g. Fig. 5 (d)) are conducted in order to compare the performance of the proposed method with EM-based [3], MRF-based [3], PCA-based [2], and GA-cluster-based [15] methods. Here, the FA and the MA are used as quantitative measurements for comparison. The results are tabulated in Table II and shown in Fig. 5. Fig. 5 (a) and (d) show the input images used by the algorithms. Fig. 5 (c) is the ground truth image. The semi-synthetic image (Fig. 5 (b)) is corrupted by increasing levels of Gaussian noise changing between $50 \mathrm{db}$ and $10 \mathrm{~dB}$ (for example see Fig. 5 (d)). According to the quantitative results of the change detection methods, EM-based change detection method provides the highest error for all considered noise levels. In the MRF-based change detection method, the FA rate is decreased by a factor of up to 3.2, but the MA rate is increased by a factor of up to 1.12. Both qualitative and quantitative results show that the EM-based and the MRF-based change detection methods give the highest rate of incorrect detection rates, even when a small amount of zero mean Gaussian noise is added. Table II and Fig. 5 show that the PCA-based change detection method has a lower rate of incorrect detection. However, in this method, the 
TABLE II: QUANTITATIVE RESULTS OF THE CHANGE DETECTION METHODS.

\begin{tabular}{|c||c|c||c|c||c|c||c|c||c|c||}
\hline \multicolumn{1}{|c||}{} & \multicolumn{2}{c||}{ EM } & \multicolumn{2}{c||}{ MRF } & \multicolumn{2}{c|}{ PCA } & \multicolumn{2}{c||}{ GA-Cluster } & \multicolumn{2}{c||}{ Proposed } \\
\hline \hline PSNR & FA & MA & FA & MA & FA & MA & FA & MA & FA & MA \\
\hline 50 & 0.09 & 0.5 & 0.03 & 0.46 & 0 & 0 & 0 & 0 & 0 & 0 \\
\hline 45 & 0.11 & 0.86 & 0.07 & 0.92 & 0.019 & 0.008 & 0.012 & 0.003 & 0 & 0 \\
\hline 40 & 1.99 & 1.42 & 1.37 & 1.65 & 0.30 & 0.73 & 0.5 & 0.21 & 0.079 & 0.051 \\
\hline 35 & 4.41 & 2.02 & 2.89 & 2.48 & 0.98 & 1.60 & 1.14 & 1.33 & 0.51 & 1.33 \\
\hline 30 & 12.31 & 6.97 & 10.3 & 7.55 & 2.72 & 4.18 & 1.71 & 4.09 & 0.89 & 2.67 \\
\hline 25 & 19.76 & 11.83 & 16.52 & 13.25 & 5.53 & 9.91 & 2.41 & 6.37 & 1.07 & 4.54 \\
\hline 20 & 30.69 & 21.78 & 20.41 & 23.06 & 14.64 & 11.11 & 3.04 & 8.18 & 1.22 & 5.79 \\
\hline 15 & 84.71 & 88.59 & 23.10 & 89.08 & 72.39 & 13.23 & 13.68 & 49.64 & 1.52 & 37.51 \\
\hline 10 & 90.24 & 93.91 & 28.08 & 92.47 & 83.71 & 16.57 & 24.07 & 62.53 & 1.92 & 49.49 \\
\hline Avg. & 27.14 & 25.32 & 11.42 & 25.66 & 20.03 & 6.37 & 5.17 & 14.71 & 0.80 & 11.27 \\
\hline
\end{tabular}

FA and the MA rates are sharply increased when the noise level is less than $25 \mathrm{~dB}$. From the results we can conclude that the proposed technique and the GA-cluster-based method provide lower error rates for all Gaussian noise levels. Comparing with the GA-cluster-based method, our method provides the highest correct detection rates.

\section{Comparing Performance of Change Detection Algorithms on Real Landsat Images}

In this experiment, the proposed method is compared with the other change detection methods which are EM-based [3], MRF-based [3], PCA-based [2], and GA-cluster-based [15] methods. Thus, we will show the robustness and effectiveness of our method comparing to the other methods. Here, to quantitatively measure the performance of the proposed change detection method, we calculate FA, MA, and TE in the region of interest.

The qualitative and quantitative results of different change detection methods on the Aral sea images (Fig. 3 (d)-(e)) are illustrated in Fig. 6 and tabulated in Table IV, respectively. Fig. 6 shows that the proposed method has the fewest isolated pixels in its corresponding binary change detection mask when compared with the results of other compared methods. It is clear from Fig. 6 and Table IV that the EM-based [3] and the MRF-based [3] change detection methods cannot give precise results. This is due to the fact that the Gaussian function is used to model the difference image which results in less accurate difference image modelling. From the results it is also obvious that the PCA-based method [2], the GA-cluster-based method [15], and the proposed method provide much more precise binary change detection masks. This improvement is achieved because the difference image itself is used to discriminant between changed and unchanged pixels. The proposed change detection method gives the lowest TE rate of $0.91 \%$, while the other compared change detection methods obtain $7.36 \%$, $6.59 \%, 3.81 \%$, and $1.69 \%$, respectively. From the results we can conclude that using the improved correlation coefficient similarity metric in chromaticity space, which makes the algorithm more robust to illumination changes, can lead to fewer isolated pixels in binary change detection mask. Another reason that our method provides more accurate results is that converting the Landsat images into grayscale images affects the process of selection of changed and unchanged pixels as different colours can have the same grayscale value.

Table III shows the quantitative results of different change detection methods on forest Landsat images (Fig. 3 (a)- (b)). The proposed change detection method gives the lowest total error rate of $1.06 \%$, while the other compared change detection methods provide $8.58 \%, 7.39 \%, 4.89 \%$, and $2.43 \%$, respectively. It is obvious from the experimental results that the binary change detection mask obtained by the proposed method is more precise when compared with those of the other methods.

\section{TABLE III: QUANTITATIVE RESULTS OBTAINING FROM DIFFERENT CHANGE DETECTION METHODS ON LANDSAT IMAGES SHOWN IN Fig. 3 (a)-(b).}

\begin{tabular}{|c||c|c|c|}
\hline Methods & $P_{F A}$ & $P_{M A}$ & $P_{T E}$ \\
\hline \hline EM & 5.90 & 21.49 & 8.58 \\
\hline MRF & 3.08 & 15.01 & 7.39 \\
\hline PCA & 2.14 & 11.74 & 4.89 \\
\hline GA-Cluster & 1.55 & 7.09 & 2.43 \\
\hline Proposed & 0.61 & 2.49 & 1.06 \\
\hline
\end{tabular}

TABLE IV: QUANTITATIVE RESULTS OBTAINING FROM DIFFERENT CHANGE DETECTION METHODS ON LANDSAT IMAGES SHOWN IN Fig. 3(d)-(e).

\begin{tabular}{|c||c|c|c|}
\hline Methods & $P_{F A}$ & $P_{M A}$ & $P_{T E}$ \\
\hline \hline EM & 5.17 & 9.70 & 7.36 \\
\hline MRF & 5.01 & 7.84 & 6.59 \\
\hline PCA & 2.48 & 5.39 & 3.81 \\
\hline GA-Cluster & 1.57 & 2.53 & 1.69 \\
\hline Proposed & 1.02 & 0.76 & 0.91 \\
\hline
\end{tabular}

\section{CONCLUSION}

In this paper, a new unsupervised approach is presented to find changed and unchanged pixels between satellite images. Our algorithm consists of two main steps. First, a preprocessing method is used to the input images to remove the illumination artefacts. The second one is to use SAPSOGA method by running the GA and PSO simultaneously to increase the probability of finding the optimal solution quickly and efficiently. By running the SAPSOGA, each algorithm informs each other by sharing information or individuals with their best results. After that, final change detection map is obtained by minimising a cost function which is based on the enhanced correlation coefficient similarity measurement. The proposed method presented in this paper minimises the change detection optimisation problem effectively to utilise local and global search capabilities of PSO and GA, respectively, to reduce the 

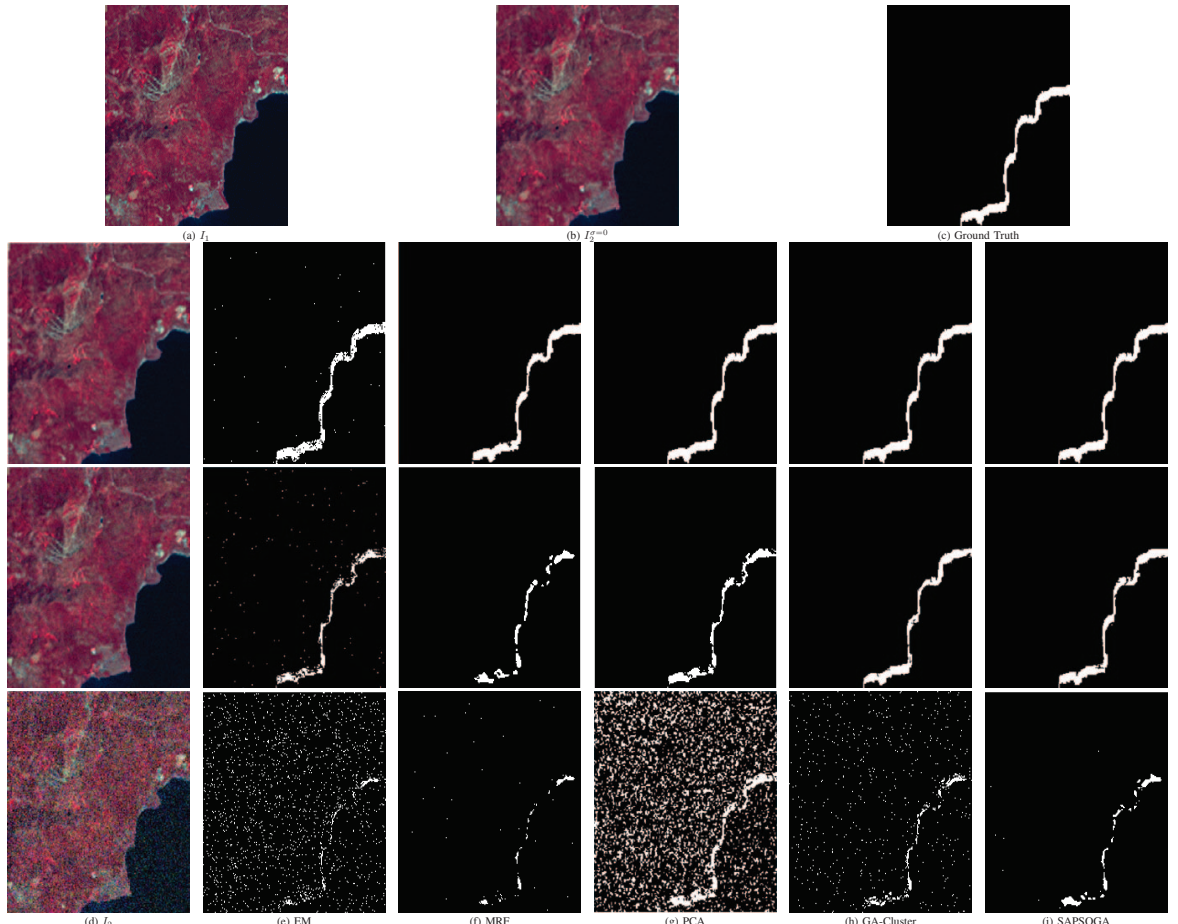

Fig. 5: Illustration of change detection results for semi-synthetic data set with different noise levels. (a): reference image $\left(X_{1}\right)$. (b): synthetic data. (c): ground truth binary image, (d), semi-synthetic image (b) noisy images corrupted by Gaussian noise (from up to down, PSNR is 45,30 , and $15 \mathrm{~dB}$, respectively). (e)-(i): result binary mask obtained by (e): EM-based, (f): MRF-based, (g): PCA-based, (h): GA-cluster-based, and (i): SAPSOGA, respectively.

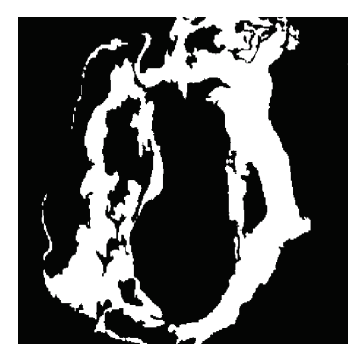

(a) Ground truth

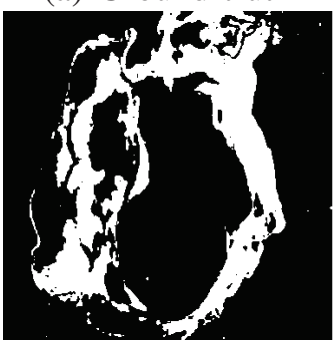

(d) PCA

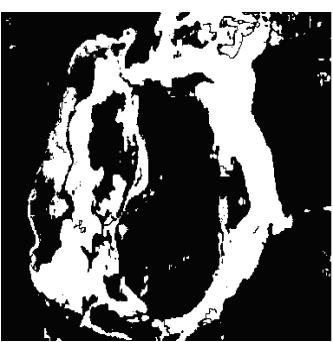

(b) EM

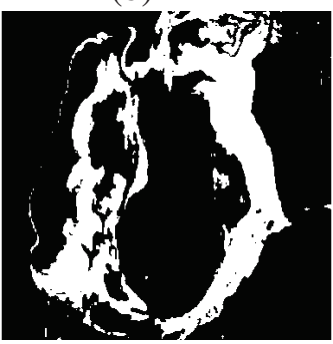

(e) GA-Cluster

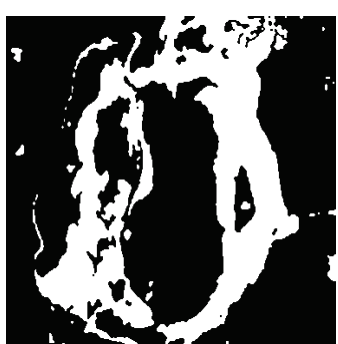

(c) MRF

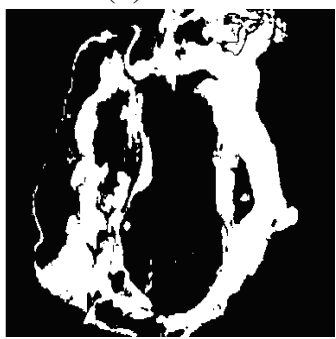

(f) Proposed

Fig. 6: Estimated binary images for the Aral sea data set.

computational burden. Qualitative and quantitative tests on two different data sets show that our method remarkably reduce the error compare to other methods.

\section{ACKNOWLEDGMENT}

Huseyin Kusetogullari is partially funded by the research project "Scalable resource-efficient systems for big data analytics" by the Knowledge Foundation (grant: 20140032) in Sweden. 


\section{REFERENCES}

[1] R. Collins, A. Lipton, T. Kanade,"Introduction to the special section on video surveillance", IEEE Trans. on Pattern Analysis and Machine Intelligent. vol. 22, no. 8, pp. 745-746, 2000.

[2] T. Celik, "Unsupervised change detection in satellite images using principal component analysis and k-means clustering". IEEE Geoscience and Remote Sensing Letters, vol. 6, no. 4, pp. 772-776,2009

[3] L. Bruzzone, D. Prieto, "Automatic analysis of the difference image for unsupervised change detection". IEEE Trans. on Geoscience and Remote Sensing. vol. 38, no. 3, pp. 1171-1182, 2000.

[4] M. BOSC, F. Heitz, J. P. Armspach, I. Namer, D. Gounot, L. Rumbach, "Automatic change detection in multimodal serial MRI: application to multiple sclerosis lesion evolution", Neuroimage, vol. 20, pp. 643-656, 2003.

[5] E. Landis, E.Nagy, D. Keane, G. Nagy, "A technique to measure $3 D$ work-of-fracture of concrete in compression", Journal of Engineering Mechanics, vol. 126, no. 6, pp. 599-605, 1999.

[6] D. Edgington, W. Dirk, K. Salamy, C. Koch, M. Risi, R. Sherlock, "Automated event detection in underwater video", In Proc. MTS/IEEE Oceans Conference, 2003.

[7] C. Y. Fang, S. W. Chen, C. S. Fuh, Automatic change detection of driving environments in a vision-based driver assistance system". IEEE Trans. on Neural Network, vol. 4, no. 3, pp. 646-657, 2003.

[8] A. Singh, "Digital Change Detection Techniques using Remotely-sensed Data", International Journal of Remote Sensing, vol. 10, pp. 989-1003, 1989.

[9] T. Habib, J. Inglada, G. Mercier, J. Chanussot, "Support vector reduction in SVM algorithm for abrupt change detection in remote sensing", Geoscience and Remote Sensing Letters, vol. 6, no. 3, pp. 660-610, 2009.

[10] M. A. Fkirin, S. M. Badwai, S. A. Mohamed, "Change detection using neural network with improvement factor in satellite images", American Journal of Environmental Sciences, vol. 5, pp. 706-713, 2009.

[11] T. Kasetkasem, P. Varshney, ”An image change detection algorithm based on Markov random field models". IEEE Trans. on Geoscience Remote Sensing, vol. 40, no. 8, pp. 1815-1823, 2002.

[12] P. Deer, P. Eklund, "Values for the fuzzy c-means classifier in change detection for remote sensing", In Proc. Int. Conference Information Processing and Management of Uncertainty, pp. 187-194, 2002.

[13] Y. Zheng, X. Zhang, B. Hou, G. Liu, "Using combined difference image and k-means clustering for SAR image change detection", IEEE Geoscience and Remote Sensing Letters, vol. 11, no.3, pp. 691-695, 2014.

[14] X. Zhang,, Z. Li, B. Hou, L. Jiao, "Spectral clustering based unsupervised change detection in SAR images, In: Proceedings of International Conference on Geoscience and Remote Sensing Symposium, pp. 712 715, 2011

[15] T. Celik, "Change detection in satellite images using a genetic algorithm approach", IEEE Geoscience and Remote Sensing Letters, vol. 7, no. 2, pp. 386-390, 2010

[16] G. D. Evangelidis, E. Z. Psarakis, "Parametric image alignment using enhanced correlation coefficient maximization", IEEE Trans. on Pattern Analysis and Machine Intelligence, vol. 30, no.10, pp. 1858-1865, 2008.

[17] J. Kennedy and R. Eberhart, "Particle Swarm Optimization", In Proc. IEEE International Conference on Neural Networks, pp. 1942-1948, 1995.

[18] H. Kusetogullari, M. S. Leeson, B. Kole, E. Hines, "Meta-heuristic algorithms for optimized network flow wavelet-based image coding", vol. 14, pp. 536-553, 2014.

[19] J. Zhang, H. S.-H. Chung, W.-L. Lo, "Clustering-Based Adaptive Crossover and Mutation Probabilities for Genetic Algorithms", IEEE Trans. on Evolutionary Computation, vol. 11, no.3, pp. 326-335, June 2007.

[20] Retrieved on August 2015 from the World Wide Web: http://geochange.er.usgs.gov/sw/changes/natural/reno-tahoe/conifer.html.

[21] Retrieved on August 2015 from the World Wide Web: http://earthengine.google.org/\#intro/AralSea. 